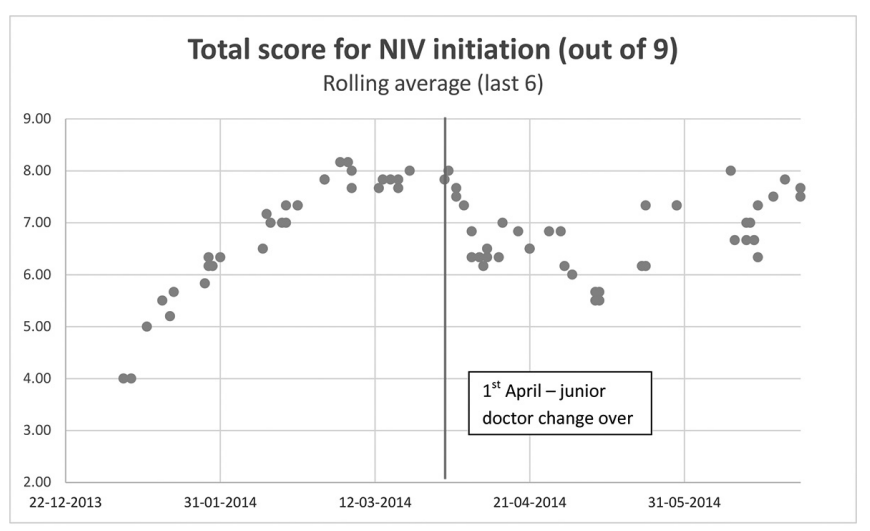

Figure 1 - Rolling average of last 6 scores - total out of 9 parameters assessed

Abstract P108 Figure 1 Rolling average of last 6 scores - total out of 9 parameters assessed

\section{P109 CAN A THEORY-INFORMED INTERACTIVE ANIMATION INCREASE INTENTIONS TO ENGAGE IN PHYSICAL ACTIVITY IN YOUNG PEOPLE WITH ASTHMA?}

${ }^{1} \mathrm{G}$ Hoskins, ${ }^{1} \mathrm{~B}$ Williams, ${ }^{2} \mathrm{~J}$ Murray, ${ }^{1} \mathrm{~S}$ Skar, ${ }^{3} \mathrm{~J}$ McGhee, ${ }^{4} \mathrm{D}$ Gauld, ${ }^{5} \mathrm{G}$ Brown, ${ }^{6} \mathrm{~S}$ Treweek, ${ }^{7} \mathrm{~F}$ Sniehotta, ${ }^{8} \mathrm{~L}$ Cameron, ${ }^{9} \mathrm{~A}$ Sheikh, ${ }^{10} \mathrm{~S}$ Hagen. ${ }^{1}$ University of Stirling, Stirling, Scotland, UK; ${ }^{2}$ Edinburgh Napier University, Edinburgh, Scotland, UK; ${ }^{3}$ University of New South Wales, Sidney, Australia; ${ }^{4}$ University of Dundee, Dundee, Scotland, UK; ${ }^{5}$ Asthma UK Scotland, Edinburgh, Scotland, UK; ${ }^{6}$ University of Aberdeen, Aberdeen, Scotland, UK; ${ }^{7}$ University of Newcastle, Newcastle, England, UK; ${ }^{8}$ University of California Merced, California, USA; ${ }^{9}$ University of Edinburgh, Edinburgh, Scotland, UK; ${ }^{10}$ Glasgow Caledonian University, Glasgow, Scotland, UK

10.1136/thoraxjnl-2014-206260.250

Background Participation in regular physical activity improves aerobic fitness and well-being. For people with asthma the benefits also include reduced hospital admissions, absenteeism, medication use, and improved ability to cope with the disease. However, although people with asthma can exercise safely, children and young people with asthma are less likely to be physically active than their peers. Integrating the principles of usercentred design and the MRC Framework for Complex interventions a theoretically-informed interactive animation was developed to encourage young people aged $12-18$ years with asthma to engage in physical activity.

Methods A mixed-methods two stage approach was used. In stage 1 a user group (young people with asthma, parents, health professionals) used online consultation and discussion methods to inform the development of the intervention in a highly iterative manner (modelling). The theoretical basis for the intervention was then refined and converted into a $3 \mathrm{D}$ animation with accompanying action plan and volitional help sheet. In stage 2 a web-based Interactive Modelling Experiment evaluated effectiveness in three key areas: knowledge about asthma, inhaler use, and intention to increase physical activity. One-to-one interviews and focus groups were used to evaluate the acceptability of the animation and whether the theoretical basis was effective.

Results Twenty three people were recruited to the user group. Facilitated by multiple online consultation methods the group was highly engaged throughout. Fifty-three individuals were randomised online to receive the intervention or control; 26 completed follow-up questionnaires (49\%). The pilot online experiment supported the evidence base for the intervention but demonstrated that recruitment methods and loss to follow-up need addressed before a future trial. Though not powered to detect an effect on intentions and behaviour, the study revealed an impact on intentions to be active (increase) and on safe inhaler use (decrease). Qualitative feedback was positive across all groups, the intervention being well received and regarded as understandable, meaningful, engaging and potentially very useful within an asthma review.

Conclusion We have developed a high quality, two part intervention regarded as meaningful, acceptable and potentially useful. Future work is needed to establish whether acceptability levels and perceived effectiveness translate into behaviour change.

\section{P110 EXPOSURE TO COMMUNITY COPD DURING SPECIALTY TRAINING}

${ }^{1} \mathrm{GH}$ Jones, ${ }^{2} \mathrm{~A}$ Collins, 'S Sibley, 'D Wat, 'MJ Walshaw. 'Liverpool Heart and Chest Hospital, Liverpool, UK; ${ }^{2}$ Royal Liverpool and Broadgreen University Hospital, Liverpool, UK

\subsection{6/thoraxjn-2014-206260.251}

Introduction There has been an exponential growth in Community COPD care delivered by respiratory consultants in the last 5 years. Despite this rapid expansion, little is known about trainees' exposure to these services even though a proportion of them will go on to work in or set up such posts after completing their training.

Methods We conducted a national survey of respiratory trainees to assess their views and experience of Community COPD, including the commissioning process which forms an integral part of establishing such services.

Results We obtained 59 responses from trainees in 12 different regions (including all four home nations): $17 \%$ less than full time (LTFT), 81\% ST5 +, and 53\% female. Despite the majority (64\%) being aware of an expansion in services locally over $86 \%$ had no experience of Community COPD and most $(75 \%)$ had no direct involvement in the commissioning process. Unsurprisingly, over three quarters felt their exposure to Community COPD services during specialty training was inadequate.

Of 8 who had attended Community COPD clinics, 7 had to organise ad hoc sessions themselves. Some trainees perceived a reluctance to facilitate formal training opportunities due to hospital service delivery requirements or a concern that community services were commissioned to be consultant-delivered.

While over $22 \%$ of respondents stated that they would not apply for a consultant post that included any community COPD sessions, this did not include any LTFT trainees and this group were also more likely to consider a job wholly based in the community [50\% vs. $27 \%$ ].

Discussion This survey suggests that most respiratory trainees, especially those in LTFT, would be willing to work in Community COPD roles as consultants, but are struggling to obtain adequate experience during training. It is envisaged that there will be further expansion of community respiratory services to improve patient access and to facilitate integration between primary and secondary care in the future. We suggest that training programme directors consider making experience of Community COPD a formal requirement and that the curriculum is updated to better reflect the needs of trainees. 